MATHEMATICS OF COMPUTATION

Volume 69, Number 229, Pages 305-323

S 0025-5718(99)01169-2

Article electronically published on August 17, 1999

\title{
EXTRAPOLATION METHODS AND DERIVATIVES OF LIMITS OF SEQUENCES
}

\author{
AVRAM SIDI
}

\begin{abstract}
Let $\left\{S_{m}\right\}$ be an infinite sequence whose limit or antilimit $S$ can be approximated very efficiently by applying a suitable extrapolation method $\mathrm{E}_{0}$ to $\left\{S_{m}\right\}$. Assume that the $S_{m}$ and hence also $S$ are differentiable functions of some parameter $\xi, \frac{d}{d \xi} S$ being the limit or antilimit of $\left\{\frac{d}{d \xi} S_{m}\right\}$, and that we need to approximate $\frac{d}{d \xi} S$. A direct way of achieving this would be by applying again a suitable extrapolation method $\mathrm{E}_{1}$ to the sequence $\left\{\frac{d}{d \xi} S_{m}\right\}$, and this approach has often been used efficiently in various problems of practical importance. Unfortunately, as has been observed at least in some important cases, when $\frac{d}{d \xi} S_{m}$ and $S_{m}$ have essentially different asymptotic behaviors as $m \rightarrow \infty$, the approximations to $\frac{d}{d \xi} S$ produced by this approach, despite the fact that they are good, do not converge as quickly as those obtained for $S$, and this is puzzling. In this paper we first give a rigorous mathematical explanation of this phenomenon for the cases in which $E_{0}$ is the Richardson extrapolation process and $\mathrm{E}_{1}$ is a generalization of it, thus showing that the phenomenon has very little to do with numerics. Following that, we propose a procedure that amounts to first applying the extrapolation method $\mathrm{E}_{0}$ to $\left\{S_{m}\right\}$ and then differentiating the resulting approximations to $S$, and we provide a thorough convergence and stability analysis in conjunction with the Richardson extrapolation process. It follows from this analysis that the new procedure for $\frac{d}{d \xi} S$ has practically the same convergence properties as $\mathrm{E}_{0}$ for $S$. We show that a very efficient way of implementing the new procedure is by actually differentiating the recursion relations satisfied by the extrapolation method used, and we derive the necessary algorithm for the Richardson extrapolation process. We demonstrate the effectiveness of the new approach with numerical examples that also support the theory. We discuss the application of this approach to numerical integration in the presence of endpoint singularities. We also discuss briefly its application in conjunction with other extrapolation methods.
\end{abstract}

\section{INTRODUCTION AND MOTIVATION}

Let $\left\{S_{m}\right\}$ be an infinite sequence with limit or antilimit $S$ that can be approximated efficiently by suitable extrapolation methods. Assume that the $S_{m}$ and hence $S$ are differentiable functions of some parameter $\xi, \frac{d}{d \xi} S$ being the limit or antilimit of $\left\{\frac{d}{d \xi} S_{m}\right\}$, and that $\frac{d}{d \xi} S_{m}$ and $S_{m}$ have essentially different asymptotic behaviors as $m \rightarrow \infty$. Suppose that we need to approximate $\frac{d}{d \xi} S$. In this paper we propose a procedure by which this can be accomplished very efficiently via derivatives of extrapolation methods used for approximating $S$.

Received by the editor March 26, 1998.

1991 Mathematics Subject Classification. Primary 40A25, 41A60, 65B05, 65B10, 65D30.

(C)1999 American Mathematical Society 
Without further delay we proceed to the motivation of this approach, which we present through the well-known Richardson extrapolation process that we use as our model. In doing so we keep the treatment general by recalling that infinite sequences are either directly related to or can be formally associated with a function $A(y)$, where $y$ may be a continuous or discrete variable.

1.1. Brief review of the Richardson extrapolation process. Let a function $A(y)$ be known and hence computable for $y \in(0, b]$ with some $b>0$, the variable $y$ being continuous or discrete. Assume, furthermore, that $A(y)$ has an asymptotic expansion of the form

$$
A(y) \sim A+\sum_{k=1}^{\infty} \alpha_{k} y^{\sigma_{k}} \text { as } y \rightarrow 0+,
$$

where $\sigma_{k}$ are known scalars satisfying

$$
\sigma_{k} \neq 0, k=1,2, \ldots ; \Re \sigma_{1}<\Re \sigma_{2}<\cdots ; \lim _{k \rightarrow \infty} \Re \sigma_{k}=+\infty,
$$

and $A$ and $\alpha_{k}, k=1,2, \ldots$, are constants independent of $y$ that are not necessarily known.

From (1.1) and (1.2) it is clear that $A=\lim _{y \rightarrow 0+} A(y)$ when this limit exists. When $\lim _{y \rightarrow 0+} A(y)$ does not exist, $A$ is the antilimit of $A(y)$ for $y \rightarrow 0+$, and in this case $\Re \sigma_{1} \leq 0$ necessarily. In any case, $A$ can be approximated very effectively by the Richardson extrapolation process that is defined via the linear systems of equations

$$
A\left(y_{l}\right)=A_{n}^{(j)}+\sum_{k=1}^{n} \bar{\alpha}_{k} y_{l}^{\sigma_{k}}, \quad j \leq l \leq j+n
$$

with the $y_{l}$ picked as

$$
y_{l}=y_{0} \omega^{l}, l=0,1, \ldots, \text { for some } y_{0} \in(0, b] \text { and } \omega \in(0,1) .
$$

Here $A_{n}^{(j)}$ are the approximations to $A$ and the $\bar{\alpha}_{k}$ are additional (auxiliary) unknowns. As is well-known, $A_{n}^{(j)}$ can be computed very efficiently by the following algorithm due to Bulirsch and Stoer [BS]:

$$
\begin{aligned}
& A_{0}^{(j)}=A\left(y_{j}\right), \quad j=0,1, \ldots, \\
& A_{n}^{(j)}=\frac{A_{n-1}^{(j+1)}-c_{n} A_{n-1}^{(j)}}{1-c_{n}}, \quad j=0,1, \ldots, n=1,2, \ldots,
\end{aligned}
$$

where we have defined

$$
c_{n}=\omega^{\sigma_{n}}, \quad n=1,2, \ldots
$$

Note that $\left\{y_{l}\right\}$ can be chosen to be an arbitrary positive decreasing sequence in $(0, b]$ such that $\lim _{l \rightarrow \infty} y_{l}=0$. Actually, $\left\{y_{l}\right\}$ can be chosen to make the extrapolation process more economical computationally. The choice of the $y_{l}$ in (1.4), however, enables us to compute the $A_{n}^{(j)}$ by the very simple and elegant algorithm in (1.5) and to give a complete analysis of convergence and stability, and hence to justify our procedure rigorously. 
1.2. The generalized Richardson extrapolation process for $\frac{d}{d \xi} A$. Let us now consider the situation in which $A(y)$ and hence $A$ depend on some real or complex parameter $\xi$ and are continuously differentiable in $\xi$ for $\xi$ in some set $X$ of the real line or the complex plane, and we are interested in computing $\frac{d}{d \xi} A \equiv \dot{A}$. Let us assume in addition to the above that $\frac{d}{d \xi} A(y) \equiv \dot{A}(y)$ has an asymptotic expansion for $y \rightarrow 0+$ that is obtained by differentiating that in (1.1) term by term. (This assumption is satisfied at least in some cases of practical interest, as can be shown rigorously). Finally, let us assume that the $\alpha_{k}$ and $\sigma_{k}$, as well as $A(y)$ and $A$, depend on $\xi$ and that they are continuously differentiable for $\xi \in X$. As a consequence of these assumptions we have

$$
\dot{A}(y) \sim \dot{A}+\sum_{k=1}^{\infty}\left(\dot{\alpha}_{k}+\alpha_{k} \dot{\sigma}_{k} \log y\right) y^{\sigma_{k}} \text { as } y \rightarrow 0+,
$$

where $\dot{\alpha}_{k} \equiv \frac{d}{d \xi} \alpha_{k}$ and $\dot{\sigma}_{k} \equiv \frac{d}{d \xi} \sigma_{k}$. Obviously, $\dot{A}$ and the $\dot{\alpha}_{k}$ and $\dot{\sigma}_{k}$ are independent of $y$. As a result, the infinite sum on the right-hand side of (1.7) is simply of the form $\sum_{k=1}^{\infty}\left(\alpha_{k 0}+\alpha_{k 1} \log y\right) y^{\sigma_{k}}$, with $\alpha_{k 0}$ and $\alpha_{k 1}$ constants independent of $y$.

Note that when the $\sigma_{k}$ do not depend on $\xi$, we have $\dot{\sigma}_{k}=0$ for all $k$, and, therefore, the asymptotic expansion in (1.7) becomes of exactly the same form as that given in (1.1). This means that we can apply the Richardson extrapolation process above directly to $\dot{A}(y)$ and obtain very good approximations to $\dot{A}$. This amounts to replacing $A\left(y_{j}\right)$ in (1.5) by $\dot{A}\left(y_{j}\right)$, keeping everything else the same. However, when the $\sigma_{k}$ are functions of $\xi$, the asymptotic expansion in (1.7) is essentially different from that in (1.1). This is so since $y^{\sigma_{k}} \log y$ and $y^{\sigma_{k}}$ behave entirely differently as $y \rightarrow 0+$. In this case the application of the Richardson extrapolation process directly to $\dot{A}(y)$ does not produce approximations to $\dot{A}$ that are of practical value.

The existence of an asymptotic expansion for $\dot{A}(y)$ of the form given in (1.7), however, suggests immediately that a generalized Richardson extrapolation process can be applied to produce approximations to $\dot{A}$ in an efficient manner. In keeping with the convention we introduced in [Si3], this extrapolation process is defined via the linear systems

$$
B\left(y_{l}\right)=B_{n}^{(j)}+\sum_{k=1}^{\lfloor(n+1) / 2\rfloor} \bar{\alpha}_{k 0} y_{l}^{\sigma_{k}}+\sum_{k=1}^{\lfloor n / 2\rfloor} \bar{\alpha}_{k 1} y_{l}^{\sigma_{k}} \log y_{l}, \quad j \leq l \leq j+n,
$$

where $B(y) \equiv \dot{A}(y), B_{n}^{(j)}$ are the approximations to $B \equiv \dot{A}$, and $\bar{\alpha}_{k 0}$ and $\bar{\alpha}_{k 1}$ are additional (auxiliary) unknowns. (This amounts to "eliminating" from (1.7) the functions $y^{\sigma_{1}}, y^{\sigma_{1}} \log y, y^{\sigma_{2}}, y^{\sigma_{2}} \log y, \ldots$, in this order.) Provided we take the $y_{l}$ as in (1.4), which is what we shall do throughout, the approximations $B_{n}^{(j)}$ can be computed very efficiently by the following algorithm developed in [Si3] and called the SGRom-algorithm there:

$$
\begin{aligned}
& B_{0}^{(j)}=B\left(y_{j}\right), \quad j=0,1, \ldots, \\
& B_{n}^{(j)}=\frac{B_{n-1}^{(j+1)}-\lambda_{n} B_{n-1}^{(j)}}{1-\lambda_{n}}, j=0,1, \ldots, n=1,2, \ldots,
\end{aligned}
$$

where we have now defined

$$
\lambda_{2 k-1}=\lambda_{2 k}=c_{k}, \quad k=1,2, \ldots,
$$

with the $c_{n}$ as defined in (1.6). 
Before going on, we would like to mention that the problem we have described above arises naturally in the numerical evaluation of integrals of the form $B=$ $\int_{0}^{1}(\log x) x^{\xi} g(x) d x$, where $\Re \xi>-1$ and $g \in C^{\infty}[0,1]$. It is easy to see that $B=\frac{d}{d \xi} A$, where $A=\int_{0}^{1} x^{\xi} g(x) d x$. Furthermore, the trapezoidal rule approximation $B(h)$ to $B$ with stepsize $h$ has an Euler-Maclaurin (E-M) expansion that is obtained by differentiating with respect to $\xi$ the E-M expansion of the trapezoidal rule approximation $A(h)$ to $A$. With this knowledge available, $B$ can be approximated by applying a generalized Richardson extrapolation process to $B(h)$. Traditionally, this approach has been adopted in multidimensional integration of singular functions as well. For a detailed discussion see Davis and Rabinowitz [DR]. See also Sidi [Si2].

1.3. Theoretical comparison of $A_{n}^{(j)}$ and $B_{n}^{(j)}$. If we arrange the $A_{n}^{(j)}$ and $B_{n}^{(j)}$ in two-dimensional arrays of the form

$$
\begin{array}{cccccc}
Q_{0}^{(0)} & & & & \\
Q_{0}^{(1)} & Q_{1}^{(0)} & & & \\
Q_{0}^{(2)} & Q_{1}^{(1)} & Q_{2}^{(0)} & & \\
Q_{0}^{(3)} & Q_{1}^{(2)} & Q_{2}^{(1)} & Q_{3}^{(0)} & \\
\vdots & \vdots & \vdots & \vdots & \ddots
\end{array}
$$

then the diagonal sequences $\left\{Q_{n}^{(j)}\right\}_{n=0}^{\infty}$ with fixed $j$ have much better convergence properties than the column sequences $\left\{Q_{n}^{(j)}\right\}_{j=0}^{\infty}$ with fixed $n$. In particular, the following convergence results are known:

1. The column sequences satisfy

$$
\begin{aligned}
& A_{n}^{(j)}-A=O\left(\left|c_{n+1}\right|^{j}\right) \text { as } j \rightarrow \infty, \\
& B_{2 m+s}^{(j)}-B=O\left(j^{1-s}\left|c_{m+1}\right|^{j}\right) \text { as } j \rightarrow \infty, s=0,1 .
\end{aligned}
$$

2. Under the additional condition that

$$
\Re \sigma_{k+1}-\Re \sigma_{k} \geq d>0, \quad k=1,2, \ldots, \text { for some fixed } d,
$$

and assuming that $\alpha_{k}, \dot{\alpha}_{k}$, and $\alpha_{k} \dot{\sigma}_{k}$ grow with $k$ at most like $\exp \left(\beta k^{\eta}\right)$ for some $\beta \geq 0$ and $\eta<2$, the diagonal sequences satisfy, for all practical purposes,

$$
\begin{aligned}
& A_{n}^{(j)}-A=O\left(\prod_{i=1}^{n}\left|c_{i}\right|\right) \text { as } n \rightarrow \infty \\
& B_{n}^{(j)}-B=O\left(\prod_{i=1}^{n}\left|\lambda_{i}\right|\right) \text { as } n \rightarrow \infty .
\end{aligned}
$$

In addition, $A_{n}^{(j)}$ and $B_{n}^{(j)}$ can also be expressed in the form

$$
A_{n}^{(j)}=\sum_{i=0}^{n} \rho_{n i} A\left(y_{j+i}\right) \text { and } B_{n}^{(j)}=\sum_{i=0}^{n} \theta_{n i} B\left(y_{j+i}\right),
$$


where both $\rho_{n i}$ and $\theta_{n i}$ are independent of $j$ and are defined through

$$
U_{n}(z) \equiv \prod_{i=1}^{n} \frac{z-c_{i}}{1-c_{i}}=\sum_{i=0}^{n} \rho_{n i} z^{i} \quad \text { and } \quad V_{n}(z) \equiv \prod_{i=1}^{n} \frac{z-\lambda_{i}}{1-\lambda_{i}}=\sum_{i=0}^{n} \theta_{n i} z^{i} .
$$

The numerical stability of $A_{n}^{(j)}$ and $B_{n}^{(j)}$ with respect to errors (computational or other) in the input values $A\left(y_{l}\right)$ and $B\left(y_{l}\right)$ is controlled by the quantities

$$
\Gamma_{n}^{(j)}=\sum_{i=0}^{n}\left|\rho_{n i}\right| \text { and } \Upsilon_{n}^{(j)}=\sum_{i=0}^{n}\left|\theta_{n i}\right|,
$$

respectively, and independently of $j$. From the fact that $\sum_{i=0}^{n} \rho_{n i}=1$ and $\sum_{i=0}^{n} \theta_{n i}$ $=1$, which results by letting $z=1$ in (1.16), we have $\Gamma_{n}^{(j)} \geq 1$ and $\Upsilon_{n}^{(j)} \geq 1$. Thus, the larger $\Gamma_{n}^{(j)}$ and $\Upsilon_{n}^{(j)}$, the worse the propagation of errors into the computed values of $A_{n}^{(j)}$ and $B_{n}^{(j)}$. We also have

$$
\Gamma_{n}^{(j)} \leq \prod_{i=1}^{n} \frac{1+\left|c_{i}\right|}{\left|1-c_{i}\right|} \text { and } \Upsilon_{n}^{(j)} \leq \prod_{i=1}^{n} \frac{1+\left|\lambda_{i}\right|}{\left|1-\lambda_{i}\right|}
$$

with equality in both cases when the $c_{i}$ all have the same phase or, equivalently, when all $\sigma_{i}$ have the same imaginary part.

The results pertaining to $A_{n}^{(j)}$ in (1.12), (1.14) and (1.18), with real $\sigma_{k}$, are due to Bulirsch and Stoer [BS]. The case of complex $\sigma_{k}$ is contained in Sidi [Si3], and so are the results on $B_{n}^{(j)}$. Actually, [Si3] gives a complete treatment of the general case in which

$$
A(y) \sim A+\sum_{k=1}^{\infty}\left[\sum_{i=0}^{q_{k}} \alpha_{k i}(\log y)^{i}\right] y^{\sigma_{k}} \quad \text { as } y \rightarrow 0+,
$$

where the $q_{k}$ are known arbitrary nonnegative integers, the $\alpha_{k i}$ are constants independent of $y$, and the $\sigma_{k}$ satisfy the condition

$$
\sigma_{k} \neq 0, k=1,2, \ldots ; \Re \sigma_{1} \leq \Re \sigma_{2} \leq \cdots ; \quad \lim _{k \rightarrow \infty} \Re \sigma_{k}=+\infty,
$$

which is much weaker than that in (1.2). Thus, the asymptotic expansions in (1.1) and (1.7) are special cases of that in (1.19) with $q_{k}=0, k=1,2, \ldots$, and $q_{k}=1, k=1,2, \ldots$, respectively.

Comparison of the diagonal sequences $\left\{A_{n}^{(j)}\right\}_{n=0}^{\infty}$ and $\left\{B_{n}^{(j)}\right\}_{n=0}^{\infty}$ (with $j$ fixed) with the help of (1.14) reveals that the latter has inferior convergence properties, even though the computational costs of $A_{n}^{(j)}$ and $B_{n}^{(j)}$ are almost identical. (They involve the computation of $A\left(y_{l}\right), l \leq j \leq j+n$, and $B\left(y_{l}\right), l \leq j \leq n$, respectively.) As a matter of fact, from (1.6), (1.10), and (1.14) it follows that the bound on $\left|A_{2 m}^{(j)}-A\right|$ is smaller than that on $\left|B_{2 m}^{(j)}-B\right|$ by a factor of $O\left(\prod_{i=1}^{m}\left|c_{m+i} / c_{i}\right|\right)=$ $O\left(\omega^{d m^{2}}\right)$ as $m \rightarrow \infty$. This theoretical observation is also supported by numerical experiments. Judging from (1.14) again, we see that, when $\Re \sigma_{k+1}-\Re \sigma_{k}=d$ for all $k$ in (1.13), $B_{\lfloor\sqrt{2} n\rfloor}^{(j)}$ will have an accuracy comparable to that of $A_{n}^{(j)}$. This, however, increases the cost of the extrapolation substantially, as the cost of computing $A\left(y_{l}\right)$ and $B\left(y_{l}\right)$ increases drastically with increasing $l$ in most cases of interest. 
1.4. A new procedure for $\frac{d}{d \xi} A$. This inferior quality of $B_{n}^{(j)}$ relative to that of $A_{n}^{(j)}$ is rather disappointing. Thus, we wonder whether we can somehow approximate $B=\dot{A}$ as accurately as we are able to approximate $A$ by $A_{n}^{(j)}$, and at a cost similar to that of $A_{n}^{(j)}$ as well. For this we first take a critical look at how the $B_{n}^{(j)}$ are determined.

Now even though the equations in (1.8) that define the $B_{n}^{(j)}$ are based directly on the asymptotic expansion of $\dot{A}(y)$ given in (1.7), it is clear that the generalized Richardson extrapolation process applied to $B(y)$ completely ignores the fact that $B(y)$ and $B$ are derivatives with respect to $\xi$ of $A(y)$ and $A$, respectively. In fact, $A(y)$ does not feature directly in (1.8) at all. Similarly, the relation of $\dot{\alpha}_{k}$ and $\dot{\sigma}_{k}$ to $\alpha_{k}$ and $\sigma_{k}$, respectively, is also ignored. These seem to be important facts to overlook.

Since we do not necessarily know either the $\alpha_{k}$ or the $\dot{\alpha}_{k}$, there is apparently nothing we can do with the fact that they are related. We do, however, know the function $A(y)$ as well as $\dot{A}(y)$. On the basis of this it can be argued that an approximation procedure that is better than the one that generates the $B_{n}^{(j)}$ should probably make use of both $\dot{A}(y)$ and $A(y)$. One such procedure may be to directly differentiate $A_{n}^{(j)}$ with respect to $\xi$ and take $\frac{d}{d \xi} A_{n}^{(j)} \equiv \dot{A}_{n}^{(j)}$ as the desired approximation to $\dot{A}$. This is exactly what we propose to do in the present work. We must add immediately that, a priori, there is no reason for $\dot{A}_{n}^{(j)}$ to approximate $\dot{A}$ as well as $A_{n}^{(j)}$ approximates $A$. In fact, we are not even sure that $\dot{A}_{n}^{(j)}$ is as good as $B_{n}^{(j)}$. In addition, we must find a way to actually compute $\dot{A}_{n}^{(j)}$. From the equations in (1.3) that define $A_{n}^{(j)}$, it is not clear how this computation is to be carried out. For this purpose, we propose to differentiate the recursion relation in (1.5) that is satisfied by $A_{n}^{(j)}$.

As far as we know, our approach is new and so is the relevant theory. Needless to say, this approach can be used for computing higher order derivatives of $A$ with respect to $\xi$. Furthermore, it can be used for computing partial derivatives of arbitrary orders of $A$ in case $A$ depends on more than one parameter.

1.5. Outline of the paper. In the next section, we develop an economical recursive algorithm for computing the $\dot{A}_{n}^{(j)}$. In Section 3 we provide some results on the $\rho_{n i}$ and $\frac{d}{d \xi} \rho_{n i}$ that are very useful in studying the stability and convergence properties of both $A_{n}^{(j)}$ and $\dot{A}_{n}^{(j)}$. In Section 4 we analyze the stability of column and diagonal sequences of the $\dot{A}_{n}^{(j)}$. The main result of this section is Theorem 4.1, which states that both column and diagonal sequences are stable. In Section 5 we dwell on the convergence issue for both $A_{n}^{(j)}$ and $\dot{A}_{n}^{(j)}$. In Theorem 5.1 we give a convergence result on column sequences that assumes (1.2), but not necessarily (1.13). In Theorem 5.2 we give computable upper bounds on $\left|A_{n}^{(j)}-A\right|$ and $\left|\dot{A}_{n}^{(j)}-\dot{A}\right|$ that we use to derive powerful convergence results for diagonal sequences. In Theorem 5.3 we show that both $A_{n}^{(j)}$ and $\dot{A}_{n}^{(j)}$ converge as $n \rightarrow \infty$ at rates faster than $\exp (-\lambda n)$ for any $\lambda>0$, assuming (1.13). (For $\dot{A}_{n}^{(j)}$ there is an additional mild assumption on the $\dot{\sigma}_{k}$.) Here the result for $A_{n}^{(j)}$ seems to be new. In Theorem 5.4 we show that, by imposing suitable growth conditions on the $\alpha_{k}$ and $\dot{\alpha}_{k}$, in addition to those already imposed on the $\sigma_{k}$ in Theorem 5.3, both $A_{n}^{(j)}$ and $\dot{A}_{n}^{(j)}$ converge as $n \rightarrow \infty$ practically at the rate of $\left(\prod_{i=1}^{n}\left|c_{i}\right|\right)$, i.e., at worst at the rate of $\exp \left(-\kappa n^{2}\right)$ 
with $\kappa=\frac{1}{2}\left(\log \omega^{-1}\right) d$, hence at the same rate as well. (The part of this theorem pertaining to $A_{n}^{(j)}$ is already given in (1.14) and follows from a more general result in [Si3], as mentioned earlier. We have chosen to give a much simpler proof of it here.) Thus, the new procedure for approximating $\dot{A}$ proposed in this paper seems to be superior to the generalized Richardson extrapolation process applied solely to $B(y) \equiv \dot{A}(y)$.

In Section 6 we give a numerical example that supports the theory of the sections preceding it. In Section 7 we show how the new approach can be applied very economically to integrals (simple or multidimensional) of functions that have logarithmic singularities at endpoints, corners, and along lines and surfaces. Finally, in Section 8 we discuss briefly how to extend the procedure of this paper to a generalized Richardson extrapolation process and other extrapolation processes.

As the developments in the remainder of this paper will involve the $c_{k}$ abundantly, before we go on it is important to make the observations that

$$
c_{k} \neq 1, k=1,2, \ldots ; \quad\left|c_{1}\right|>\left|c_{2}\right|>\cdots ; \lim _{k \rightarrow \infty} c_{k}=0 .
$$

These follow from (1.2) and (1.6).

Also, note that all the developments of this paper, including the recursive algorithm of the next section and the theory of the subsequent sections, directly apply to infinite sequences $\left\{S_{m}\right\}$ that satisfy

$$
S_{m} \sim S+\sum_{k=1}^{\infty} \beta_{k} c_{k}^{m} \text { as } m \rightarrow \infty
$$

with the $c_{k}$ as in (1.21). Here we have the analogy $S_{m} \leftrightarrow A\left(y_{m}\right)$, and $S \leftrightarrow A$, the rest remaining the same.

\section{ReCURSive COMPUtATION OF $\dot{A}_{n}^{(j)}$}

Differentiating the recursion relation given in (1.5), and invoking (1.5) again, we obtain the following recursive algorithm:

$$
\begin{aligned}
& A_{0}^{(j)}=A\left(y_{j}\right) \text { and } \dot{A}_{0}^{(j)}=\dot{A}\left(y_{j}\right), j=0,1, \ldots, \\
& A_{n}^{(j)}=\frac{A_{n-1}^{(j+1)}-c_{n} A_{n-1}^{(j)}}{1-c_{n}}, \\
& \dot{A}_{n}^{(j)}=\frac{\dot{A}_{n-1}^{(j+1)}-c_{n} \dot{A}_{n-1}^{(j)}}{1-c_{n}}+\frac{\dot{c}_{n}}{1-c_{n}}\left(A_{n}^{(j)}-A_{n-1}^{(j)}\right), j=0,1, \ldots, n=1,2, \ldots .
\end{aligned}
$$

Here $\dot{c}_{n} \equiv \frac{d}{d \xi} c_{n}, n=1,2, \ldots$. This shows that we need two tables of the form given in (1.11), one for $A_{n}^{(j)}$ and another for $\dot{A}_{n}^{(j)}$. We also see that the computation of the $\dot{A}_{n}^{(j)}$ involves both $\dot{A}(y)$ and $A(y)$.

As is clear from (2.1), the computation of $\dot{A}_{n}^{(j)}$ involves $A\left(y_{l}\right)$ and $\dot{A}\left(y_{l}\right), l=$ $j, j+1, \ldots, j+n$, whereas that of $A_{n}^{(j)}$ involves only $A\left(y_{l}\right), l=j, j+1, \ldots, j+n$. Thus it may be argued that the computational cost of $\dot{A}_{n}^{(j)}$ is twice that of $A_{n}^{(j)}$. However, at least in some problems of practical interest, such as those that arise in numerical integration, $\dot{A}(y)$ can be computed simultaneously with $A(y)$ and at almost no additional cost. In other words, the computational cost of $\dot{A}_{n}^{(j)}$ for such 
cases is practically the same as that of $A_{n}^{(j)}$. This is a very important and useful feature of the method that we have proposed.

\section{Theoretical PRELIMINARIES}

Let us recall the definitions of $c_{n}$ in (1.6), of $\rho_{n i}$ in (1.15), and the result pertaining to $\sum_{i=0}^{n} \rho_{n i} z^{i}$ in (1.16). Theorem 3.1 below is a special case of Theorem 2.1 in $[\mathrm{Si} 3]$.

Theorem 3.1. The $\rho_{n i}$ satisfy

$$
X_{n}(z) \equiv \sum_{i=0}^{n}\left|\rho_{n i}\right||z|^{i} \leq \prod_{i=1}^{n} \frac{|z|+\left|c_{i}\right|}{\left|1-c_{i}\right|} \equiv \tilde{X}_{n}(z) .
$$

Corollary. Assume the condition on the $\sigma_{k}$ given in (1.13). Then there exist positive constants $L_{0}, L_{1}, \ldots$, and $M$, all independent of $n$, for which

$$
\begin{gathered}
X_{n}(1)=\sum_{i=0}^{n}\left|\rho_{n i}\right|<L_{0}, \\
X_{n}\left(c_{s+1}\right)=\sum_{i=0}^{n}\left|\rho_{n i}\right|\left|c_{s+1}\right|^{i}<L_{s}\left|c_{s+1}\right|^{n}, \quad n \geq s,
\end{gathered}
$$

and

$$
X_{n}\left(c_{n+1}\right)=\sum_{i=0}^{n}\left|\rho_{n i}\right|\left|c_{n+1}\right|^{i} \leq M\left(\prod_{i=1}^{n}\left|c_{i}\right|\right) .
$$

Proof. We first note that the condition in (1.13) implies that $\left|c_{i+1} / c_{i}\right| \leq \omega^{d}$ for all $i$. To prove (3.2) we let $z=1$ in (3.1). We thus obtain

$$
\sum_{i=0}^{n}\left|\rho_{n i}\right| \leq \prod_{i=1}^{n} \frac{1+\left|c_{i}\right|}{\left|1-c_{i}\right|}<\prod_{i=1}^{\infty} \frac{1+\left|c_{i}\right|}{\left|1-c_{i}\right|} \equiv L_{0} .
$$

The infinite products in (3.5) converge and have nonzero limits, since the series $\sum_{i=1}^{\infty} c_{i}$ converges absolutely by the fact that $\left|c_{i+1} / c_{i}\right| \leq \omega^{d}<1$ and by the ratio test. To prove (3.3) we let $z=c_{s+1}$ in (3.1). We then have

$$
\sum_{i=0}^{n}\left|\rho_{n i}\right|\left|c_{s+1}\right|^{i} \leq\left|c_{s+1}\right|^{n}\left(\prod_{i=1}^{n} \frac{1+\left|c_{i} / c_{s+1}\right|}{\left|1-c_{i}\right|}\right) .
$$

The result follows again by the fact that the infinite products $\prod_{i=1}^{\infty}\left|1-c_{i}\right|$ and $\prod_{i=1}^{\infty}\left(1+\left|c_{i} / c_{s+1}\right|\right)$ converge and have nonzero limits. Finally, to prove (3.4) we let $z=c_{n+1}$ in (3.1). We have

$$
\sum_{i=0}^{n}\left|\rho_{n i}\right|\left|c_{n+1}\right|^{i} \leq\left(\prod_{i=1}^{n}\left|c_{i}\right|\right)\left(\prod_{i=1}^{n} \frac{1+\left|c_{n+1} / c_{i}\right|}{\left|1-c_{i}\right|}\right) \leq\left(\prod_{i=1}^{n}\left|c_{i}\right|\right)\left(\prod_{i=1}^{n} \frac{1+\omega^{d i}}{\left|1-c_{i}\right|}\right) .
$$

The proof can be completed as before.

Theorem 3.2. Define $\dot{\rho}_{n i} \equiv \frac{d}{d \xi} \rho_{n i}$ and $\dot{c}_{i} \equiv \frac{d}{d \xi} c_{i}$. Then

$$
Y_{n}(z) \equiv \sum_{i=0}^{n}\left|\dot{\rho}_{n i}\right||z|^{i} \leq\left(\sum_{i=1}^{n} b_{i}(z)\right) \tilde{X}_{n}(z),
$$


where $\tilde{X}_{n}(z)$ is the upper bound on $X_{n}(z)$ given in (3.1) and

$$
b_{i}(z)=\left(\frac{1}{\left|1-c_{i}\right|}+\frac{1}{|z|+\left|c_{i}\right|}\right)\left|\dot{c}_{i}\right|, i=1,2, \ldots .
$$

Proof. We start with the recursion relation

$$
\rho_{n i}=\frac{\rho_{n-1, i-1}-c_{n} \rho_{n-1, i}}{1-c_{n}}, i=0,1 \ldots, n,
$$

where we adopt the convention $\rho_{n i}=0$ for $i<0$ and $i>n$. Differentiating both sides of (3.10), and invoking (3.10) again, we obtain

$$
\dot{\rho}_{n i}=\frac{\dot{\rho}_{n-1, i-1}-c_{n} \dot{\rho}_{n-1, i}}{1-c_{n}}+\frac{\dot{c}_{n}}{1-c_{n}}\left(\rho_{n i}-\rho_{n-1, i}\right), i=0,1, \ldots, n .
$$

Taking moduli on both sides of (3.11), multiplying by $|z|^{i}$ and summing over $i$, we obtain

$$
Y_{n}(z) \leq \frac{|z|+\left|c_{n}\right|}{\left|1-c_{n}\right|} Y_{n-1}(z)+\frac{\left|\dot{c}_{n}\right|}{\left|1-c_{n}\right|}\left[X_{n}(z)+X_{n-1}(z)\right]
$$

which, by invoking the upper bounds $\tilde{X}_{n}(z)$ and $\tilde{X}_{n-1}(z)$ on $X_{n}(z)$ and $X_{n-1}(z)$, respectively, becomes

$$
Y_{n}(z) \leq \frac{|z|+\left|c_{n}\right|}{\left|1-c_{n}\right|} Y_{n-1}(z)+b_{n}(z) \tilde{X}_{n}(z) .
$$

Starting now with the fact that $Y_{0}(z) \equiv 0$ and using induction on $n$, the result in (3.8) follows.

Corollary. Assume the condition on the $\sigma_{k}$ given in (1.13). Assume also that $\sum_{i=1}^{\infty}\left|\dot{c}_{i}\right|<\infty$. Then there exist positive constants $L_{0}^{\prime}, L_{1}^{\prime}, \ldots$, independent of $n$, such that

$$
Y_{n}(1)=\sum_{i=0}^{n}\left|\dot{\rho}_{n i}\right|<L_{0}^{\prime}
$$

and

$$
Y_{n}\left(c_{s+1}\right)=\sum_{i=0}^{n}\left|\dot{\rho}_{n i}\right|\left|c_{s+1}\right|^{i}<L_{s}^{\prime}\left|c_{s+1}\right|^{n}, \quad n \geq s .
$$

If also $\left|\dot{c}_{i}\right| \leq K_{i}\left|c_{i}\right|, i=1,2, \ldots$, for some $K_{i}>0$, then there exists $M^{\prime}>0$, independent of $n$, such that

$$
Y_{n}\left(c_{n+1}\right)=\sum_{i=0}^{n}\left|\dot{\rho}_{n i}\right|\left|c_{n+1}\right|^{i}<\left(M^{\prime}+\sum_{i=1}^{n} K_{i}\right)\left(\prod_{i=1}^{n}\left|c_{i}\right|\right) .
$$

Proof. The proof can be achieved by setting $z=1, z=c_{s+1}$, and $z=c_{n+1}$ in (3.8), and recalling that $\lim _{n \rightarrow \infty} c_{n}=0$. Thus, $b_{i}(1) \sim 2\left|\dot{c}_{i}\right|$ as $i \rightarrow \infty, b_{i}\left(c_{s+1}\right) \sim$ $\left(1+\left|c_{s+1}\right|^{-1}\right)\left|\dot{c}_{i}\right|$ as $i \rightarrow \infty$, and $b_{i}\left(c_{n+1}\right)<\left|1-c_{i}\right|^{-1}\left|\dot{c}_{i}\right|+K_{i} \sim\left|\dot{c}_{i}\right|+K_{i}$ as $i \rightarrow \infty$, independently of $n$. In particular, we have $L_{0}^{\prime}=\left[\sum_{i=1}^{\infty} b_{i}(1)\right] L_{0}<\infty$. We leave the details to the redear.

A very commonly occurring case is one in which $K_{i} \leq K$ for all $i$. In such a case $\sum_{i=1}^{n} K_{i} \leq K n$. When $K_{i}=O\left(i^{-1}\right)$ as $i \rightarrow \infty$, then $\sum_{i=1}^{n} K_{i}=O(\log n)$ as $n \rightarrow \infty$. When $K_{i}=O\left(i^{a}\right)$ as $i \rightarrow \infty, a>-1$, then $\sum_{i=1}^{n} K_{i}=O\left(n^{a+1}\right)$ as $n \rightarrow \infty$. In all these cases $\sum_{i=1}^{n} K_{i} \rightarrow \infty$ at worst like $n^{\tilde{a}}$ with some $\tilde{a}>0$, and, furthermore, we have $\sum_{i=1}^{\infty}\left|\dot{c}_{i}\right|<\infty$, since $\left|c_{i}\right|=\omega^{\Re \sigma_{i}} \leq \omega^{\Re \sigma_{1}-d} \omega^{i d}$ and $\omega \in(0,1)$. 


\section{Stability}

In Section 1 we mentioned the well-known fact that $\Gamma_{n}^{(j)}=\sum_{i=0}^{n}\left|\rho_{n i}\right|$, cf. (1.17), controls the propagation of errors in input values of $A\left(y_{l}\right)$ into $A_{n}^{(j)}$ by the fact that $A_{n}^{(j)}=\sum_{i=0}^{n} \rho_{n i} A\left(y_{j+i}\right)$, cf. (1.15). Analogously, by the fact that

$$
\dot{A}_{n}^{(j)}=\sum_{i=0}^{n} \rho_{n i} \dot{A}\left(y_{j+i}\right)+\sum_{i=0}^{n} \dot{\rho}_{n i} A\left(y_{j+i}\right)
$$

what controls the propagation of errors in input values of $A\left(y_{l}\right)$ and $\dot{A}\left(y_{l}\right)$ into $\dot{A}_{n}^{(j)}$ is now

$$
\Omega_{n}^{(j)}=\sum_{i=0}^{n}\left|\rho_{n i}\right|+\sum_{i=0}^{n}\left|\dot{\rho}_{n i}\right|=X_{n}(1)+Y_{n}(1)
$$

independently of $j$. Using (3.1), we can bound $X_{n}(1)$ by $\tilde{X}_{n}(1)$, obtaining the easily computable bound on $\Gamma_{n}^{(j)}$ given already in (1.18). Using also (3.13), we can bound $Y_{n}(1)$ by $\tilde{Y}_{n}(1)$, where $\tilde{Y}_{n}(z)$ are determined from the recursion

$$
\tilde{Y}_{n}(z)=\frac{|z|+\left|c_{n}\right|}{\left|1-c_{n}\right|} \tilde{Y}_{n-1}(z)+b_{n}(z) \tilde{X}_{n}(z), \quad n=1,2, \ldots ; \quad \tilde{Y}_{0}(z)=0 .
$$

Substituting these in (4.2), we obtain

$$
\Omega_{n}^{(j)} \leq \tilde{X}_{n}(1)+\tilde{Y}_{n}(1),
$$

which provides us with an easily computable bound for $\Omega_{n}^{(j)}$.

The following theorem summarizes the subject of stability of the $\dot{A}_{n}^{(j)}$.

Theorem 4.1. The column sequences $\left\{\dot{A}_{n}^{(j)}\right\}_{j=0}^{\infty}$ (n fixed) are stable in the sense that $\sup _{j} \Omega_{n}^{(j)}<\infty$. Under the condition on the $\sigma_{k}$ given in (1.13) and the additional condition of Theorem 3.2 that $\sum_{i=1}^{\infty}\left|\dot{c}_{i}\right|<\infty$, the diagonal sequences $\left\{\dot{A}_{n}^{(j)}\right\}_{n=0}^{\infty}$ (j fixed) are stable in the sense that $\sup _{n} \Omega_{n}^{(j)}<\infty$.

Proof. The stability of column sequences follows from the fact that $\Omega_{n}^{(j)}$ is independent of $j$. The stability of diagonal sequences follows from $X_{n}(1)<L_{0}$ and $Y_{n}(1)<L_{0}^{\prime}$ in the corollaries to Theorems 3.1 and 3.2 , respectively.

\section{Convergence}

5.1. Convergence of column sequences. With the subject of stability treated, we now turn to convergence. We start with the column sequences.

Theorem 5.1. Assume that the $\sigma_{k}$ satisfy (1.2), but not necessarily (1.13). Then, for fixed $n$, the error $\dot{A}_{n}^{(j)}-\dot{A}$ has the complete asymptotic expansion

$$
\dot{A}_{n}^{(j)}-\dot{A} \sim \sum_{k=n+1}^{\infty}\left\{\frac{d}{d \xi}\left[U_{n}\left(c_{k}\right) \alpha_{k}\right]+U_{n}\left(c_{k}\right) \alpha_{k} \dot{\sigma}_{k} \log y_{j}\right\} y_{j}^{\sigma_{k}} \quad \text { as } \quad j \rightarrow \infty,
$$

as a result of which

$$
\dot{A}_{n}^{(j)}-\dot{A}=O\left(j\left|c_{n+1}\right|^{j}\right) \quad \text { as } j \rightarrow \infty .
$$


Proof. The expansion in (5.1) is obtained by term-by-term differentiation with respect to $\xi$ of the well-known asymptotic expansion of $A_{n}^{(j)}-A$, namely,

$$
A_{n}^{(j)}-A \sim \sum_{k=n+1}^{\infty} U_{n}\left(c_{k}\right) \alpha_{k} y_{j}^{\sigma_{k}} \text { as } j \rightarrow \infty .
$$

This is allowed by our assumption that $\dot{A}(y)$ has an asymptotic expansion as $y \rightarrow$ $0+$ that is obtained by term-by-term differentiation of (1.1) with respect to $\xi$. For the sake of completeness, we recall that (5.3) follows by substituting (1.1) in $A_{n}^{(j)}=\sum_{i=0}^{n} \rho_{n i} A\left(y_{j+i}\right)$ (which is justified since $\lim _{j \rightarrow \infty} y_{j}=0$ ), and then invoking $y_{j+i}=y_{j} \omega^{i}, c_{k}=\omega^{\sigma_{k}},(1.16)$, and finally $U_{n}\left(c_{k}\right)=0, k=1, \ldots, n$.

The result in (5.2) means that if the $(n-1)$ st column of the extrapolation table for $\dot{A}_{n}^{(j)}$ converges, then the $n$th column converges at least as quickly as the $(n-1)$ st column. The $n$th column converges if $\left|c_{n}\right|<1$ even when the $(n-1)$ st column diverges. If the $n$th column diverges, it does so at most as quickly as the $(n-1)$ st column does. In summary, each column is at least as good as the column preceding it. Finally, this convergence is linear.

5.2. Convergence of diagonal sequences. We next treat the convergence of the diagonal sequences. We start this by deriving upper bounds on $\left|A_{n}^{(j)}-A\right|$ and $\left|\dot{A}_{n}^{(j)}-\dot{A}\right|$ that are valid for all $j$ and $n$.

Theorem 5.2. Define for each $s$

$$
R_{s}(y)=A(y)-A-\sum_{k=1}^{s} \alpha_{k} y^{\sigma_{k}}
$$

and also

$$
\hat{\alpha}_{s+1}=\max _{0 \leq y \leq y_{0}}\left|R_{s}(y) / y^{\sigma_{s+1}}\right|
$$

and

$$
\hat{\beta}_{s+1}=\max _{0 \leq y \leq y_{0}}\left|\dot{R}_{s}(y) /\left(y^{\sigma_{s+1}} \log y\right)\right| .
$$

Then, for $n \geq s$, the errors in $A_{n}^{(j)}$ and $\dot{A}_{n}^{(j)}$ satisfy, respectively,

$$
\left|A_{n}^{(j)}-A\right| \leq \hat{\alpha}_{s+1}\left|y_{j}^{\sigma_{s+1}}\right| X_{n}\left(c_{s+1}\right)
$$

and

$$
\left|\dot{A}_{n}^{(j)}-\dot{A}\right| \leq \hat{\alpha}_{s+1}\left|y_{j}^{\sigma_{s+1}}\right| Y_{n}\left(c_{s+1}\right)+\hat{\beta}_{s+1}\left|y_{j}^{\sigma_{s+1}}\right|\left(\max _{0 \leq i \leq n}\left|\log y_{j+i}\right|\right) X_{n}\left(c_{s+1}\right) .
$$

Proof. We start with

$$
A_{n}^{(j)}-A=\sum_{i=0}^{n} \rho_{n i} R_{s}\left(y_{j+i}\right) \text { for } s \leq n,
$$

which follows by substituting (5.4) in $A_{n}^{(j)}=\sum_{i=0}^{n} \rho_{n i} A\left(y_{j+i}\right)$, and invoking (1.16), $\sum_{i=0}^{n} \rho_{n i}=1$, and $U_{n}\left(c_{k}\right)=0, k=1, \ldots, n$. Differentiating (5.9) with respect to 
$\xi$, we obtain

$$
\dot{A}_{n}^{(j)}-\dot{A}=\sum_{i=0}^{n} \dot{\rho}_{n i} R_{s}\left(y_{j+i}\right)+\sum_{i=0}^{n} \rho_{n i} \dot{R}_{s}\left(y_{j+i}\right),
$$

with $\dot{R}_{s}(y) \equiv \frac{d}{d \xi} R_{s}(y)$. From (1.1) and (1.7), we have

$$
R_{s}(y) \sim \sum_{k=s+1}^{\infty} \alpha_{k} y^{\sigma_{k}} \text { as } y \rightarrow 0+
$$

and

$$
\dot{R}_{s}(y) \sim \sum_{k=s+1}^{\infty}\left(\dot{\alpha}_{k}+\alpha_{k} \dot{\sigma}_{k} \log y\right) y^{\sigma_{k}} \text { as } y \rightarrow 0+,
$$

which guarantee the existence of finite $\hat{\alpha}_{s+1}$ and $\hat{\beta}_{s+1}$ as defined by (5.5) and (5.6), respectively. To prove (5.7) and (5.8), we now take moduli on both sides of (5.9) and (5.10), use the fact that

$$
\left|R_{s}(y)\right| \leq \hat{\alpha}_{s+1}\left|y^{\sigma_{s+1}}\right| \text { and }\left|\dot{R}_{s}(y)\right| \leq \hat{\beta}_{s+1}\left|y^{\sigma_{s+1}} \log y\right| \text { for } y \in\left(0, y_{0}\right],
$$

and then invoke $y_{j+i}=y_{j} \omega^{i}, c_{k}=\omega^{\sigma_{k}}$, and (3.1) and (3.8).

It is worth noting that when $\hat{\alpha}_{s+1}$ and $\hat{\beta}_{s+1}$ or upper bounds on them are available, (5.7) and (5.8) provide computable bounds on $\left|A_{n}^{(j)}-A\right|$ and $\left|\dot{A}_{n}^{(j)}-\dot{A}\right|$, respectively.

The bounds of Theorem 5.2 can be turned into powerful convergence theorems for the diagonals as we show next.

Theorem 5.3. (i) If (1.13) is satisfied, then

$$
A_{n}^{(j)}-A=O\left(\omega^{\mu n}\right) \text { as } n \rightarrow \infty \text {, for any } \mu>0 .
$$

(ii) If, in addition, $\sum_{i=1}^{\infty}\left|\dot{c}_{i}\right|<\infty$, then

$$
\dot{A}_{n}^{(j)}-\dot{A}=O\left(\omega^{\mu n}\right) \text { as } n \rightarrow \infty, \text { for any } \mu>0 .
$$

Proof. Invoking (3.3) in (5.7), we obtain

$$
A_{n}^{(j)}-A=O\left(\left|c_{s+1}\right|^{n}\right)=O\left(\omega^{\left(\Re \sigma_{s+1}\right) n}\right) \text { as } n \rightarrow \infty .
$$

Similarly, invoking (3.3) and (3.15) in (5.8), and noting that $\max _{0 \leq i \leq n}\left|\log y_{j+i}\right| \sim$ $|\log \omega| n$ as $n \rightarrow \infty$, we obtain

$$
\dot{A}_{n}^{(j)}-\dot{A}=O\left(n\left|c_{s+1}\right|^{n}\right)=O\left(n \omega^{\left(\Re \sigma_{s+1}\right) n}\right) \text { as } n \rightarrow \infty .
$$

The results in (5.14) and (5.15) now follow if we realize that $s$ in (5.16) and (5.17) is arbitrary and that $\lim _{k \rightarrow \infty} \Re \sigma_{k}=+\infty$.

What Theorem 5.3 implies is that the diagonal sequences $\left\{A_{n}^{(j)}\right\}_{n=0}^{\infty}$ and $\left\{\dot{A}_{n}^{(j)}\right\}_{n=0}^{\infty}$ all converge and do so superlinearly in the sense that $\left|A_{n}^{(j)}-A\right|$ and $\left|\dot{A}_{n}^{(j)}-\dot{A}\right|$ tend to 0 as $n \rightarrow \infty$ like $\exp (-\lambda n)$ for any $\lambda>0$. By imposing suitable growth conditions on the $\hat{\alpha}_{k}$ and $\hat{\beta}_{k}$, we next show that $\left|A_{n}^{(j)}-A\right|$ and $\left|\dot{A}_{n}^{(j)}-\dot{A}\right|$ tend to 0 like $\exp \left(-\kappa n^{2}\right)$ for some $\kappa>0$. 
Theorem 5.4. (i) In part (i) of Theorem 5.3 assume also that $\hat{\alpha}_{k} y_{0}^{\sigma_{k}}=O\left(e^{\beta k^{\eta}}\right)$ as $k \rightarrow \infty$, for some $\beta \geq 0$ and $\eta<2$. Then, for any $\varepsilon>0$ such that $\omega+\varepsilon<1$,

$$
A_{n}^{(j)}-A=O\left((\omega+\varepsilon)^{d n^{2} / 2}\right) \text { as } n \rightarrow \infty .
$$

(ii) In part (ii) of Theorem 5.3 assume also that both $\hat{\alpha}_{k} y_{0}^{\sigma_{k}}=O\left(e^{\beta k^{\eta}}\right)$ and $\hat{\beta}_{k} y_{0}^{\sigma_{k}}=O\left(e^{\beta k^{\eta}}\right)$ as $k \rightarrow \infty$ for some $\beta \geq 0$ and $\eta<2$. In addition, assume that $\left|\dot{c}_{i}\right| \leq K_{i}\left|c_{i}\right|, i=1,2 \ldots$, with $K_{i}=O\left(i^{a}\right)$ as $i \rightarrow \infty, a \geq-1$. Then, for any $\varepsilon>0$ such that $\omega+\varepsilon<1$,

$$
\dot{A}_{n}^{(j)}-\dot{A}=O\left((\omega+\varepsilon)^{d n^{2} / 2}\right) \text { as } n \rightarrow \infty .
$$

Proof. Letting $s=n$ in (5.7) and (5.8), and invoking (3.4) and (3.16), we obtain, respectively,

$$
\left|A_{n}^{(j)}-A\right| \leq M \hat{\alpha}_{n+1}\left|y_{j}^{\sigma_{n+1}}\right|\left(\prod_{i=1}^{n}\left|c_{i}\right|\right)
$$

and

$$
\begin{aligned}
\mid \dot{A}_{n}^{(j)} & -\dot{A} \mid \\
& \leq\left[\left(M^{\prime}+\sum_{i=1}^{n} K_{i}\right) \hat{\alpha}_{n+1}+M\left(\max _{0 \leq i \leq n}\left|\log y_{j+i}\right|\right) \hat{\beta}_{n+1}\right]\left|y_{j}^{\sigma_{n+1}}\right|\left(\prod_{i=1}^{n}\left|c_{i}\right|\right) .
\end{aligned}
$$

The results can be obtained by noting that

$$
\prod_{i=1}^{n}\left|c_{i}\right|=\omega^{\sum_{i=1}^{n} \Re \sigma_{i}} \leq \omega^{\left(\Re \sigma_{1}\right) n+d n(n-1) / 2},
$$

which follows from (1.13), and $\sum_{i=1}^{n} K_{i}=O\left(n^{\tilde{a}}\right)$ as $n \rightarrow \infty$ for some $\tilde{a} \geq 0$, and

$$
\max _{0 \leq i \leq n}\left|\log y_{j+i}\right| \sim|\log \omega| n \text { as } n \rightarrow \infty,
$$

which follows from (1.6). We leave the details to the reader.

Note. Under the conditions of Theorem 5.4 it is clear from (5.21) that both $\left|A_{n}^{(j)}-A\right|$ and $\left|\dot{A}_{n}^{(j)}-\dot{A}\right|$ tend to 0 as $n \rightarrow \infty$ at the rate of $\left(\prod_{i=1}^{n}\left|c_{i}\right|\right)$ for all practical purposes.

\section{A NUMERICAL EXAMPLE}

In this section we shall apply our new procedure to the summation of the infinite series $\sum_{k=1}^{\infty}(-\log k) k^{-\xi-1}$, whether this series converges or not. For $\Re \xi>0$ the series converges and represents a function analytic for $\Re \xi>0$ in the $\xi$-plane. Furthermore, this function is $\zeta^{\prime}(\xi+1)=\frac{d}{d \xi} \zeta(\xi+1)$, where $\zeta(\xi+1)$ is the Riemann zeta function defined by the convergent infinite series $\sum_{k=1}^{\infty} k^{-\xi-1}$ for $\Re \xi>0$ and then continued analytically to the rest of the $\xi$-plane. Obviously, if we let $\sum_{k=1}^{m} k^{-\xi-1}=A_{m}$, then $\sum_{k=1}^{m}(-\log k) k^{-\xi-1}=\frac{d}{d \xi} A_{m} \equiv \dot{A}_{m}$. It is known that, whether $\left\{A_{m}\right\}$ converges or not, and as long as $\xi \neq 0$,

$$
A_{n} \sim \zeta(\xi+1)+\sum_{k=1}^{\infty} \alpha_{k} n^{-\xi-k+1} \text { as } n \rightarrow \infty
$$


with $\alpha_{1}=-\xi^{-1}, \alpha_{2}=1 / 2, \alpha_{k}=-\xi^{-1}\left(\begin{array}{c}-\xi \\ k-1\end{array}\right) B_{k-1}, k=3,4, \ldots$. Here $B_{i}$ are the Bernoulli numbers. It can also be shown that $\frac{d}{d \xi} A_{n}=\dot{A}_{n}$ has an asymptotic expansion as $n \rightarrow \infty$ that is obtained by differentiating the one in (6.1) termwise. Thus,

$$
\dot{A}_{n} \sim \zeta^{\prime}(\xi+1)+\sum_{k=1}^{\infty}\left(\dot{\alpha}_{k}-\alpha_{k} \log n\right) n^{-\xi-k+1} \quad \text { as } n \rightarrow \infty .
$$

By drawing the analogy $A_{n} \leftrightarrow A(y), n^{-1} \leftrightarrow y, \zeta(\xi+1) \leftrightarrow A, \sigma_{k}=\xi+k-1, k=$ $1,2, \ldots$, we see that $A(y)$ is of the form discussed throughout the paper, provided $\xi \neq 0,-1,-2, \ldots$. Also, $y$ now is a discrete variable that takes on the values $1,1 / 2,1 / 3, \ldots$. Finally, $\dot{\sigma}_{k}=1$, and hence $\dot{c}_{k}=(\log \omega) c_{k}$ for all $k$, so that $\sum_{i=1}^{\infty}\left|\dot{c}_{i}\right|<\infty$ and $K_{i}=|\log \omega|$ for all $i$ in Theorems 5.3 and 5.4.

In our numerical experiments we took $y_{0}=1$ and $\omega=1 / 2$, so that $y_{l}=2^{-l}$ and $A\left(y_{l}\right)=A_{2^{l}}, l=0,1, \ldots$.

We computed $A_{n}^{(j)}, \dot{A}_{n}^{(j)}$, and $B_{n}^{(j)}$ for $\xi=1$ and $\xi=-1+10 \mathrm{i}$, and we give the results of the computation in Tables 6.1 and 6.2. For $\xi=1,\left\{A_{m}\right\}$ converges and has $\zeta(2)=\pi^{2} / 6$ as its limit. For $\xi=-1+10 \mathrm{i}$, it diverges with antilimit $\zeta(10 \mathrm{i})$. Similarly, $\left\{\dot{A}_{m}\right\}$ has $\zeta^{\prime}(2)$ as its limit for $\xi=1$ and $\zeta^{\prime}(10 \mathrm{i})$ as its antilimit for $\xi=-1+10 \mathrm{i}$. Note also that when $\xi=-1+10 \mathrm{i}$, the partial sum $A_{2^{n}}$ diverges like $2^{n}$ as $n \rightarrow \infty$. All our computations were done in quadruple precision arithmetic.

The results shown in Tables 6.1 and 6.2 are in complete agreement with the theory provided in the paper. In particular, $\dot{A}_{n}^{(0)}$ and $A_{n}^{(0)}$ seem to converge to their respective limits or antilimits at the same rate. Also, since $\Re \sigma_{k+1}-\Re \sigma_{k}=1$ for all $k, \dot{A}_{n}^{(0)}$ and $B_{\lfloor\sqrt{2} n\rfloor}^{(0)}$ do have comparable accuracies, in agreement with the last paragraph of subsection 1.3.

TABLE 6.1. Results of computation with the series $\sum_{k=1}^{\infty} k^{-\xi-1}$ and $\sum_{k=1}^{\infty}(-\log k) k^{-\xi-1}$ for $\xi=1$ (convergent series).

\begin{tabular}{||c||c|c||c|c|c||}
\hline$n$ & $\left|A_{2^{n}}-A\right|$ & $\left|A_{n}^{(0)}-A\right|$ & $\left|\dot{A}_{2^{n}}-\dot{A}\right|$ & $\left|\dot{A}_{n}^{(0)}-\dot{A}\right|$ & $\left|B_{n}^{(0)}-B\right|$ \\
\hline \hline 0 & $6.45 D-01$ & $6.45 D-01$ & $9.38 D-01$ & $9.38 D-01$ & $9.38 D-01$ \\
\hline 1 & $3.95 D-01$ & $1.45 D-01$ & $7.64 D-01$ & $2.44 D-01$ & $5.91 D-01$ \\
\hline 2 & $2.21 D-01$ & $1.53 D-02$ & $5.56 D-01$ & $3.01 D-02$ & $1.03 D-01$ \\
\hline 3 & $1.18 D-01$ & $5.16 D-04$ & $3.69 D-01$ & $1.14 D-03$ & $9.10 D-03$ \\
\hline 4 & $6.06 D-02$ & $8.13 D-06$ & $2.30 D-01$ & $2.71 D-05$ & $4.25 D-03$ \\
\hline 5 & $3.08 D-02$ & $4.43 D-07$ & $1.38 D-01$ & $1.49 D-06$ & $3.98 D-05$ \\
\hline 6 & $1.55 D-02$ & $4.74 D-10$ & $8.01 D-02$ & $7.81 D-10$ & $2.42 D-05$ \\
\hline 7 & $7.78 D-03$ & $4.28 D-11$ & $4.56 D-02$ & $1.94 D-10$ & $7.55 D-07$ \\
\hline 8 & $3.90 D-03$ & $8.79 D-14$ & $2.55 D-02$ & $4.15 D-13$ & $6.82 D-09$ \\
\hline 9 & $1.95 D-03$ & $3.92 D-16$ & $1.41 D-02$ & $2.26 D-15$ & $1.47 D-09$ \\
\hline 10 & $9.76 D-04$ & $4.79 D-19$ & $7.74 D-03$ & $2.92 D-18$ & $4.45 D-11$ \\
\hline 11 & $4.88 D-04$ & $3.01 D-22$ & $4.21 D-03$ & $2.11 D-21$ & $2.51 D-14$ \\
\hline 12 & $2.44 D-04$ & $1.88 D-25$ & $2.27 D-03$ & $1.38 D-24$ & $6.09 D-15$ \\
\hline 13 & $1.22 D-04$ & $1.66 D-29$ & $1.22 D-03$ & $1.39 D-28$ & $4.23 D-17$ \\
\hline 14 & $6.10 D-05$ & $1.58 D-32$ & $6.53 D-04$ & $6.07 D-32$ & $6.25 D-19$ \\
\hline
\end{tabular}


TABLE 6.2. Results of computation with the series $\sum_{k=1}^{\infty} k^{-\xi-1}$ and $\sum_{k=1}^{\infty}(-\log k) k^{-\xi-1}$ for $\xi=-1+10 \mathrm{i}$ (divergent series).

\begin{tabular}{||c||c|c||c|c|c||}
\hline$n$ & $\left|A_{2^{n}}-A\right|$ & $\left|A_{n}^{(0)}-A\right|$ & $\left|\dot{A}_{2^{n}}-\dot{A}\right|$ & $\left|\dot{A}_{n}^{(0)}-\dot{A}\right|$ & $\left|B_{n}^{(0)}-B\right|$ \\
\hline \hline 0 & $7.63 D-01$ & $7.63 D-01$ & $4.95 D-01$ & $4.95 D-01$ & $4.95 D-01$ \\
\hline 1 & $5.04 D-01$ & $1.44 D+00$ & $4.80 D-01$ & $5.91 D-01$ & $9.94 D-01$ \\
\hline 2 & $5.54 D-01$ & $2.90 D+00$ & $8.30 D-01$ & $3.92 D+00$ & $1.57 D+00$ \\
\hline 3 & $8.97 D-01$ & $2.54 D+00$ & $1.91 D+00$ & $5.34 D+00$ & $2.54 D+00$ \\
\hline 4 & $1.67 D+00$ & $8.80 D-01$ & $4.66 D+00$ & $2.40 D+00$ & $3.83 D+00$ \\
\hline 5 & $3.25 D+00$ & $1.54 D-01$ & $1.13 D+01$ & $5.11 D-01$ & $2.89 D+00$ \\
\hline 6 & $6.42 D+00$ & $1.26 D-02$ & $2.67 D+01$ & $4.91 D-02$ & $2.19 D+00$ \\
\hline 7 & $1.28 D+01$ & $5.68 D-04$ & $6.20 D+01$ & $2.55 D-03$ & $8.00 D-01$ \\
\hline 8 & $2.55 D+01$ & $1.27 D-05$ & $1.41 D+02$ & $6.43 D-05$ & $2.58 D-01$ \\
\hline 9 & $5.10 D+01$ & $1.55 D-07$ & $3.18 D+02$ & $8.79 D-07$ & $3.95 D-02$ \\
\hline 10 & $1.02 D+02$ & $1.00 D-09$ & $7.06 D+02$ & $6.27 D-09$ & $6.10 D-03$ \\
\hline 11 & $2.04 D+02$ & $3.28 D-12$ & $1.55 D+03$ & $2.26 D-11$ & $4.98 D-04$ \\
\hline 12 & $4.08 D+02$ & $6.31 D-15$ & $3.39 D+03$ & $4.72 D-14$ & $3.51 D-05$ \\
\hline 13 & $8.15 D+02$ & $5.45 D-18$ & $7.34 D+03$ & $4.43 D-17$ & $1.38 D-06$ \\
\hline 14 & $1.63 D+03$ & $3.14 D-21$ & $1.58 D+04$ & $2.74 D-20$ & $5.36 D-08$ \\
\hline
\end{tabular}

Finally, all of these approximations can be computed quite stably. Actually, from (1.18) and (4.4), we have that $\Gamma_{n}^{(j)} \leq 9, \Upsilon_{n}^{(j)} \leq 69$, and $\Omega_{n}^{(j)} \leq 22$ for $\xi=1$, while for $\xi=-1+10$ i we have $\Gamma_{n}^{(j)} \leq 38, \Upsilon_{n}^{(j)} \leq 1387$, and $\Omega_{n}^{(j)} \leq 200$.

\section{Application to NUmerical integration}

Let us consider the numerical approximation of the integral

$$
B=\int_{0}^{1}(\log x) x^{\xi} g(x) d x, \quad \Re \xi>-1,
$$

with $g \in C^{\infty}[0,1]$. As mentioned in Section $1, B=\frac{d}{d \xi} A \equiv \dot{A}$, where $A=$ $\int_{0}^{1} x^{\xi} g(x) d x$. Let us set $h=1 / n$, where $n$ is a positive integer, and define the trapezoidal rule approximations with stepsize $h$ to $A$ and $B$, respectively, by

$$
A(h)=h\left[\sum_{j=1}^{n-1} G(j h)+\frac{1}{2} G(1)\right], \quad G(x) \equiv x^{\xi} g(x),
$$

and

$$
B(h)=h\left[\sum_{j=1}^{n-1} H(j h)+\frac{1}{2} H(1)\right], \quad H(x) \equiv(\log x) x^{\xi} g(x) .
$$

Note that $B(h)=\dot{A}(h)$ since $H(x)=\dot{G}(x)$. The following extensions of the classical Euler-Maclaurin expansion are special cases of those given by Navot in [N1] and [N2]:

$$
A(h) \sim A+\sum_{i=1}^{\infty} a_{i} h^{2 i}+\sum_{i=0}^{\infty} b_{i} h^{\xi+i+1} \quad \text { as } \quad h \rightarrow 0,
$$


and

$$
B(h) \sim B+\sum_{i=1}^{\infty} \dot{a}_{i} h^{2 i}+\sum_{i=0}^{\infty}\left(\dot{b}_{i}+b_{i} \log h\right) h^{\xi+i+1} \text { as } h \rightarrow 0,
$$

with

$$
a_{i}=\frac{B_{2 i}}{(2 i) !} G^{(2 i-1)}(1), i=1,2, \ldots ; \quad b_{i}=\frac{\zeta(-\xi-i)}{i !} g^{(i)}(0), i=0,1, \ldots,
$$

and $\dot{a}_{i}=\frac{d}{d \xi} a_{i}$ and $\dot{b}_{i}=\frac{d}{d \xi} b_{i}$. As before, $B_{k}$ are the Bernoulli numbers. The expansion in (7.4) is obtained by differentiating that in (7.3). (Note that $G(x)$ depends on $\xi$ but $g(x)$ does not.) For a different derivation of (7.3) see Lyness and Ninham [LN].

Let us now consider the case $-1<\Re \xi<0$. Then $A(h)$ is of the form described in Section 1 and treated throughout, with $\sigma_{1}, \sigma_{2}, \ldots$ as in

$$
\sigma_{3 i-2}=\xi+2 i-1, \sigma_{3 i-1}=\xi+2 i, \sigma_{3 i}=2 i, i=1,2, \ldots,
$$

so that (1.13) is satisfied with $d=\min (-\Re \xi, 1+\Re \xi)>0$.

Let us also apply the Richardson extrapolation process to the sequence $\left\{A\left(h_{l}\right)\right\}$ with $h_{l}=\omega^{l}, l=0,1, \ldots$, for some $\omega \in\{1 / 2,1 / 3, \ldots\}$. (We should, of course, keep in mind that other more economical choices of $\left\{h_{l}\right\}$ are possible, but they do not enable us to make rigorous statements about convergence, convergence rates, and stability. We therefore stick with the above choice of $\left\{h_{l}\right\}$.)

Recall now that $\left|A_{n}^{(j)}-A\right|$ is practically $O\left(\prod_{i=1}^{n}\left|c_{i}\right|\right)$ as $n \rightarrow \infty$. This implies that $\left|A_{3 m}^{(j)}-A\right| \rightarrow 0$ as $m \rightarrow \infty$ practically like $\omega^{\Sigma_{m}}$, where $\Sigma_{m}=\sum_{i=1}^{3 m} \Re \sigma_{i}=$ $3 m^{2}+O(m)$ as $m \rightarrow \infty$. (Recall the note at the end of Section 5.)

Since $\dot{\sigma}_{3 i-2}=\dot{\sigma}_{3 i-1}=1, \dot{\sigma}_{3 i}=0, i=1,2, \ldots$, we have $\dot{c}_{3 i-2}=(\log \omega) c_{3 i-2}$, $\dot{c}_{3 i-1}=(\log \omega) c_{3 i-1}, \quad \dot{c}_{3 i}=0, \quad i=1,2, \ldots$, and thus $\sum_{i=1}^{\infty}\left|\dot{c}_{i}\right|<\infty$ and $K_{i} \leq$ $|\log \omega|$ for all $i$. Consequently, Theorems 5.3 and 5.4 apply, and so $\left|\dot{A}_{3 m}^{(j)}-\dot{A}\right| \rightarrow 0$ as $m \rightarrow \infty$ like $\omega^{3 m^{2}}$ practically. (Again, recall the note at the end of Section 5.)

Similarly, $B(h)=\dot{A}(h)$ is of the form given in (1.19) with $\sigma_{i}$ as in (7.6) and $q_{3 i-2}=q_{3 i-1}=1, q_{3 i}=0, i=1,2, \ldots$. Let us also apply the generalized Richardson extrapolation process to the sequence $\left\{B\left(h_{l}\right)\right\}$ with $h_{l}=\omega^{l}, l=0,1, \ldots$, for some $\omega \in\{1 / 2,1 / 3, \ldots\}$. By Theorem 4.2 in [Si3] the sequence $\left\{B_{n}^{(j)}\right\}_{n=0}^{\infty}$ converges to $B$, and especially from equation (4.16) in the proof of this theorem, it follows that $\left|B_{5 m}^{(j)}-B\right|$ is practically $O\left(\prod_{i=1}^{3 m}\left|c_{i}\right|^{q_{i}+1}\right)$ as $m \rightarrow \infty$. This implies that $\left|B_{5 m}^{(j)}-B\right| \rightarrow 0$ as $m \rightarrow \infty$ practically like $\omega^{\Lambda_{m}}$, where $\Lambda_{m}=\sum_{i=1}^{3 m}\left(q_{i}+1\right)\left(\Re \sigma_{i}\right)=$ $5 m^{2}+O(m)$ as $m \rightarrow \infty$.

Thus, $\left|\dot{A}_{n}^{(j)}-\dot{A}\right|$ and $\left|B_{n}^{(j)}-B\right|$ tend to 0 as $n \rightarrow \infty$ like $\omega^{n^{2} / 3}$ and $\omega^{n^{2} / 5}$, respectively, for all practical purposes. That is to say, of the two diagonal sequences $\left\{\dot{A}_{n}^{(j)}\right\}_{n=0}^{\infty}$ and $\left\{B_{n}^{(j)}\right\}_{n=0}^{\infty}$ the former has superior convergence properties. Also, $B_{\lfloor\sqrt{5 / 3} n\rfloor}^{(j)}$ will have an accuracy comparable to that of $\dot{A}_{n}^{(j)}$. Of course, $B_{\lfloor\sqrt{5 / 3} n\rfloor}^{(j)}$ is much more expensive to compute than $\dot{A}_{n}^{(j)}$ itself. (Recall that the computation of $A\left(y_{l}\right)$ or $B\left(y_{l}\right)$ involves $2^{l}$ integrand evaluations.)

The comparative study above suggests, therefore, that the computation of integrals of the form $\int_{0}^{1}(\log x) x^{\xi} g(x) d x$ by first applying the Richardson extrapolation 
process to the integral $\int_{0}^{1} x^{\xi} g(x) d x$ and then differentiating the resulting approximations with respect to $\xi$ may be a preferred method if we intend to use extrapolation methods in the first place. This approach may be used in multidimensional integration of integrands that have logarithmic corner, or surface, or line singularities, for which appropriate extensions of the Euler-Maclaurin expansion can be found in, e.g., Lyness [L], Lyness and Monegato [LM], and Sidi [Si1]. All of these E-M expansions are obtained by term-by-term differentiation of other simpler E-M expansions, and this is what makes the approach of this paper appropriate. Since the computation of the trapezoidal rule approximations for multidimensional integrals becomes very expensive as the dimension increases, the economy that can be achieved by this approach should make it especially attractive.

The new approach can also be used in the computation of the singular integrals $I_{r}=\int_{0}^{1}(\log x)^{r} x^{\xi} g(x) d x$, where $r=2,3, \ldots$, by realizing that $I_{r}=\frac{d^{r}}{d \xi^{r}} A$. (Note that $B=I_{1}$.) The approximations produced by the generalized Richardson extrapolation process have convergence properties that deteriorate in quality as $r$ becomes large, whereas the $\frac{d^{r}}{d \xi^{r}} A_{n}^{(j)}$ maintain the high-quality convergence properties of the $A_{n}^{(j)}$. For the application of the generalized Richardson extrapolation process to such integrals, see [Si3]. Again, the extension to multidimensional singular integrals is immediate.

Before closing this section we would like to make the following interesting observation that is analogous to an observation of Bauer, Rutishauser, and Stiefel [BRS] about Romberg integration, see also [DR]: The approximation $\dot{A}_{n}^{(j)}$ to $B$ can be expressed as a sort of "numerical quadrature formula" with stepsize $h_{j+n}$ of the form

$$
\dot{A}_{n}^{(j)}=\sum_{k=0}^{\nu^{j+n}} w_{j n k}^{(0)} H\left(k h_{j+n}\right)+\sum_{k=0}^{\nu^{j+n}} w_{j n k}^{(1)} G\left(k h_{j+n}\right), \quad \nu=1 / \omega,
$$

in which the "weights" $w_{j n k}^{(0)}$ and $w_{j n k}^{(1)}$ depend on $j$ and $n$, and satisfy

$$
\sum_{k=0}^{\nu^{j+n}} w_{j n k}^{(0)}=1 \text { and } \sum_{k=0}^{\nu^{j+n}} w_{j n k}^{(1)}=0 .
$$

These follow from the facts that $\sum_{i=0}^{n} \rho_{n i}=1$ and $\sum_{i=0}^{n} \dot{\rho}_{n i}=0$. (We have obtained (7.7) and (7.8) by adding the terms $\frac{1}{2} G(0) h$ and $\frac{1}{2} H(0) h$ to the right-hand sides of (7.1) and (7.2), respectively, with the understanding that $G(0) \equiv 0$ and $H(0) \equiv 0$.) Also, these formulas are stable numerically, in the sense that

$$
\sum_{k=0}^{\nu^{j+n}}\left(\left|w_{j n k}^{(0)}\right|+\left|w_{j n k}^{(1)}\right|\right) \leq \Omega_{n}^{(j)} \leq L_{0}+L_{0}^{\prime}<\infty \text { for all } j \text { and } n .
$$

\section{EXtension to Other EXtRAPOLATiOn MEthods}

The derivative approach that we have introduced in Section 1 for $A(y)$ as in (1.1) and (1.2), and for $A_{n}^{(j)}$ as defined via (1.3) and (1.4), can be extended to the more general case in which

$$
A(y) \sim A+\sum_{k=1}^{\infty} \alpha_{k} \phi_{k}(y) \text { as } y \rightarrow 0+
$$


where $\left\{\phi_{k}(y)\right\}_{k=1}^{\infty}$ is a known asymptotic sequence, i.e.,

$$
\phi_{k+1}(y)=o\left(\phi_{k}(y)\right) \text { as } y \rightarrow 0+, \quad k=1,2, \ldots .
$$

In this case approximations to $A$ can be obtained through the generalized Richardson extrapolation process, which is now defined by the linear systems

$$
A\left(y_{l}\right)=A_{n}^{(j)}+\sum_{k=1}^{n} \bar{\alpha}_{k} \phi_{k}\left(y_{l}\right), \quad j \leq l \leq j+n,
$$

where $\left\{y_{l}\right\}_{l=0}^{\infty} \subset(0, b]$ is a positive decreasing sequence with $\lim _{l \rightarrow \infty} y_{l}=0$ that is picked by the user. As before, if $A(y)$ and $A$ depend on $\xi$ and $\dot{A}$ is desired, we propose to approximate $\dot{A}$ by $\dot{A}_{n}^{(j)}$, and to obtain the $\dot{A}_{n}^{(j)}$ by differentiating the recursive algorithms by which the $A_{n}^{(j)}$ are computed. Two such algorithms are the E-algorithm of Schneider [Sc], different derivations of which can be found in Håvie [H] and Brezinski [B2], and the FS-algorithm of Ford and Sidi [FS]. For arbitrary $\phi_{k}(y)$ these algorithms are quite involved, and their derivatives are even more involved. Nevertheless, this approach may yield better approximations than the generalized Richardson extrapolation process applied to the asymptotic expansion

$$
\dot{A}(y) \sim A+\sum_{k=1}^{\infty} \dot{\alpha}_{k} \phi_{k}(y)+\sum_{k=1}^{\infty} \alpha_{k} \dot{\phi}_{k}(y) \text { as } y \rightarrow 0+,
$$

and defined through

$$
B\left(y_{l}\right)=B_{n}^{(j)}+\sum_{k=1}^{\lfloor(n+1) / 2\rfloor} \bar{\alpha}_{k 0} \phi_{k}\left(y_{l}\right)+\sum_{k=1}^{\lfloor n / 2\rfloor} \bar{\alpha}_{k 1} \dot{\phi}_{k}\left(y_{l}\right), \quad j \leq l \leq j+n,
$$

assuming, of course, that $\left\{\dot{\phi}_{k}(y)\right\}_{k=1}^{\infty}$ is an asymptotic sequence as $y \rightarrow 0+$ just like $\left\{\phi_{k}(y)\right\}_{k=1}^{\infty}$, and the two are of essentially different nature.

Before closing we would like to note that the procedure of this paper can be applied in a very simple fashion in conjunction with extrapolation methods that are already defined via recursion relations, whether these are generalized Richardson extrapolation processes or not. One such method is the transformation of Shanks [Sh], which can be implemented very efficiently by the $\epsilon$-algorithm of Wynn [W]. When this transformation is applied to a sequence $\left\{S_{m}\right\}$ that depends on a parameter $\xi$, our derivative approach produces the following recursive algorithm:

$$
\begin{aligned}
& \epsilon_{-1}^{(n)}=0, \quad \dot{\epsilon}_{-1}^{(n)}=0, \quad \epsilon_{0}^{(n)}=S_{n}, \quad \text { and } \dot{\epsilon}_{0}^{(n)}=\dot{S}_{n}, \quad n=0,1, \ldots, \\
& \epsilon_{k+1}^{(n)}=\epsilon_{k-1}^{(n+1)}+\frac{1}{\epsilon_{k}^{(n+1)}-\epsilon_{k}^{(n)}}, \\
& \dot{\epsilon}_{k+1}^{(n)}=\dot{\epsilon}_{k-1}^{(n+1)}-\frac{\dot{\epsilon}_{k}^{(n+1)}-\dot{\epsilon}_{k}^{(n)}}{\left(\epsilon_{k}^{(n+1)}-\epsilon_{k}^{(n)}\right)^{2}}, \quad n=0,1, \ldots, \quad k=1,2, \ldots .
\end{aligned}
$$

Here $\epsilon_{2 k}^{(n)}$ and $\dot{\epsilon}_{2 k}^{(n)}$ are approximations to $S$, the limit or antilimit of $\left\{S_{m}\right\}$, and to $\frac{d}{d \xi} S$, respectively. Another such method is the $\theta$-algorithm of Brezinski [B1], which is defined exclusively by a recursion relation similar to the $\epsilon$-algorithm.

We propose to consider the application of the procedure of this paper to some of the known extrapolation methods in a future publication. 


\section{ACKNOWLEDGMENT}

This reserach was supported in part by the Fund for the Promotion of Research at the Technion.

\section{REFERENCES}

[BRS] F.L. Bauer, H. Rutishauser, and E. Stiefel, New aspects in numerical quadrature, in Experimental Arithmetic, High Speed Computing, and Mathematics, Proc. Sympos. Appl. Math., vol. 15, AMS, Providence, Rhode Island, 1963, pp.199-218. MR 30:4384

[B1] C. Brezinski, Accélération de suites à convergence logarithmique, C.R. Acad. Sci. Paris, 273 (1971), pp. A727-A730. MR 46:4674

[B2] C. Brezinski, A general extrapolation algorithm, Numer. Math., 35 (1980), pp. 175-187. MR 81j:65015

[BS] R. Bulirsch and J. Stoer, Fehlerabschätzungen und Extrapolation mit rationalen Funktionen bei Verfahren vom Richardson-Typus, Numer. Math., 6 (1964), pp. 413-427. MR 31:861

[DR] P.J. Davis and P. Rabinowitz, Methods of Numerical Integration, 2nd edition, Academic Press, New York, 1984. MR 86d:65004

[FS] W.F. Ford and A. Sidi, An algorithm for a generalization of the Richardson extrapolation process, SIAM J. Numer. Anal., 24 (1987), pp. 1212-1232. MR 89a:65006

[H] T. Håvie, Generalized Neville type extrapolation schemes, BIT, 19 (1979), pp. 204-213. MR 80f: 65005

[L] J.N. Lyness, An error functional expansion for $N$-dimensional quadrature with an integrand function singular at a point, Math. Comp., 30 (1976), pp. 1-23. MR 53:11976

[LM] J.N. Lyness and G. Monegato, Quadrature error functional expansions for the simplex when the integrand function has singularities at vertices, Math. Comp., 34 (1980), pp. 213-225. MR 80m:65017

[LN] J.N. Lyness and B.W. Ninham, Numerical quadrature and asymptotic expansions, Math. Comp., 21 (1967), pp. 162-178. MR 37:1081

[N1] I. Navot, An extension of the Euler-Maclaurin summation formula to functions with a branch singularity, J. Math. and Phys., 40 (1961), pp. 271-276. MR 25:4290

[N2] I. Navot, A further extension of the Euler-Maclaurin summation formula, J. Math. and Phys., 41 (1962), pp. 155-163.

[Sc] C. Schneider, Vereinfachte Rekursionen zur Richardson-Extrapolation in Spezialfällen, $\mathrm{Nu}$ mer. Math., 24 (1975), pp. 177-184. MR 51:14510

[Sh] D. Shanks, Non-linear transformations of divergent and slowly convergent sequences, $J$. Math. and Phys., 34 (1955), pp. 1-42. MR 16:961c

[Si1] A. Sidi, Euler-Maclaurin expansions for integrals over triangles and squares of functions having algebraic/logarithmic singularities along an edge, J. Approx. Th., 39 (1983), pp. 39-53. MR 84j:65026

[Si2] A. Sidi, Generalizations of Richardson extrapolation with applications to numerical integration, in Numerical Integration III, ISNM Vol. 85, H. Brass and G. Hämmerlin, eds., Birkhäuser, Basel, Switzerland, 1988, pp. 237-250. MR 90k:65076

[Si3] A. Sidi, A complete convergence and stability theory for a generalized Richardson extrapolation process, SIAM J. Numer. Anal., 34 (1997), pp. 1761-1778. MR 98g:65012

[W] P. Wynn, On a device for computing the $e_{m}\left(S_{n}\right)$ transformation, Math. Comp., 10 (1956), pp. 91-96. MR 18:801e

Computer Science Department, Technion-Israel Institute of Technology, Haifa 32000 , ISRAEL

E-mail address: asidi@cs.technion.ac.il 